\title{
The Use of All-You-Can-Drink System, Nomihodai, Is Associated with the Increased Alcohol Consumption among College Students: A Cross-Sectional Study in Japan
}

\author{
Kyoko Kawaida, ${ }^{1,2}$ Hisashi Yoshimoto,${ }^{3}$ Ryohei Goto, ${ }^{3}$ Go Saito, ${ }^{4}$ Yasukazu Ogai, ${ }^{5}$ \\ Nobuaki Morita ${ }^{5}$ and Tamaki Saito ${ }^{5}$ \\ ${ }^{1}$ Faculty of Psychiatry and Mental Health Nursing, National Defense Medical College, Tokorozawa, Saitama, \\ Japan \\ ${ }^{2}$ Graduate School of Social Psychiatry and Mental Health, Faculty of Medicine, University of Tsukuba, Tsukuba, \\ Ibaraki, Japan \\ ${ }^{3}$ Department of Primary Care and Medical Education, Faculty of Medicine, University of Tsukuba, Tsukuba, \\ Ibaraki, Japan \\ ${ }^{4}$ Primary Care and Medical Education, Graduate School of Comprehensive Human Sciences, University of \\ Tsukuba, Tsukuba, Ibaraki, Japan \\ ${ }^{5}$ Department of Social Psychiatry and Mental Health, Faculty of Medicine, University of Tsukuba, Tsukuba, \\ Ibaraki, Japan
}

Excessive drinking by college students is a major public health problem in Japan. However, data on heavy episodic drinking (HED) and nomihodai, a several-hour all-you-can-drink system, are scarce. We surveyed the drinking behavior of undergraduate and graduate students at 35 colleges, and examined the association between HED and use of nomihodai. The study used a cross-sectional design conducted by a selfadministered questionnaire. From December 2016 to March 2017, we sampled undergraduate and graduate students aged 20 or older at 35 colleges in the Kanto area, including Tokyo. The following items were measured: 1) frequency of drinking; 2) frequency of binge drinking in the past year; 3) nomihodai use; 4 ) the number of drinks consumed when using or not using nomihodai; and 5) sex and age for demographic data. Paired t-test was used to compare means between use and non-use state of nomihodai. The Fisher's exact test was used to evaluate the significance of the distribution difference between the two types of states. A total of 511 subjects completed the questionnaire, including 274 men and 237 women. The amount of drinking was increased 1.8-fold $(85.9 \pm 49.7 \mathrm{~g}$ vs. $48.2 \pm 29.5 \mathrm{~g})$ among men and 1.7-fold $(63.7 \pm 39.3 \mathrm{~g}$ vs. $36.5 \pm 26.7 \mathrm{~g})$ among women during nomihodai use, comapred with non-use states. Among them, $109(39.8 \%)$ men and $71(30.3 \%)$ women reported HED only at nomihodai states. These data suggest that the use of nomihodai system may lead to excessive drinking among college students.

Keywords: blood alcohol concentration; college student; excessive alcohol use; heavy episodic drinking; nomihodai Tohoku J. Exp. Med., 2018 August, 245 (4), 263-267. C 2018 Tohoku University Medical Press

\section{Introduction}

Excessive drinking in young people is a major public health problem worldwide. Excessive drinking not only causes acute alcohol poisoning, but also contributes to dangerous behaviors while in the drunken state (Eaton et al. 2010; World Health Organization 2014). The World Health Organization (WHO) announced that " 2.5 million people die from alcohol-related problems worldwide, including 320,000 young people aged 15 to 29" (WHO 2009). Among young people, college students consume more alcohol than non-college students (Hingson et al. 2002). A great deal of research has focused on excessive drinking by college students, and has revealed that many students experience negative alcohol-related consequences such as injury (Hingson et al. 2009; Kowalenko et al. 2013), physical assault, and drunk driving (Hingson et al. 2009). There are several types of excessive alcohol use, including heavy episodic drinking (HED) and binge drinking (White et al. 2013). HED is defined as consumption of pure alcohol intake of $60 \mathrm{~g}$ or more in one drinking occasion (WHO 2014), whereas binge drinking refers to consumption of five or more drinks (for men) or four or more drinks (for women) over a period of about two hours (National Institute of Alcohol Abuse and Alcoholism 2004). Both are dangerous drinking patterns that lead to alcohol intoxication.

Received March 27, 2018; revised and accepted July 30, 2018. Published online August 29, 2018; doi: 10.1620/tjem.245.263.

Correspondence: Hisashi Yoshimoto, Department of Primary Care and Medical Education, Faculty of Medicine, University of Tsukuba,

1-1-1 Tennoudai, Tsukuba, Ibaraki 305-8575, Japan.

e-mail: hisashiyoshi@md.tsukuba.ac.jp 
In Japan, alcohol consumption by young people aged 20 to 29 has gradually increased since 1984 (Higuchi et al. 2004), and HED and binge drinking have been observed, especially in young people (Osaki et al. 2016). Among Japanese college students, $56.8 \%$ of men and $47.8 \%$ of women are binge drinkers, and binge drinkers had a history of significantly more alcohol-related injuries (odds ratio 25.6) (Yoshimoto et al. 2017). Despite emerging evidence of alcohol-related problems of young people, including college students, there are no restrictions on sponsorship and no legislative provisions for alcohol-free environments (Higuchi et al. 2007). Moreover, there is a unique and common system, nomihodai, which enables the customer to drink various kinds of drinks within two to three hours at a fixed price. A previous study reported that "all-you-candrink" services increase the risk of binge drinking 2.44-fold (Carlini et al. 2014) and that a set price for unlimited drinking is associated with HED among college students (Wechsler et al. 2000). Also, a significant relationship has been reported between "all-you-can-drink" and blood alcohol concentration (BAC) (Thombs et al. 2009). These results were all obtained in college students, implying that college students tend to consume excessive amounts of alcohol (resulting in high BAC) under circumstances like nomihodai. Policies that restrict "flat rate for unlimited drinking," which is synonymous for "all-you-can-drink," can decrease harmful drinking (WHO 2009). Recently, "all-you-can-drink" offers were outlawed in countries such as Northern Ireland and England, which instituted fines for establishments with "all-you-can-drink" offers. On the other hand, the proportion of izakaya (Japanese bar) establishments that provide "all-you-can-drink" services is approximately $34 \%$ of all alcohol-serving establishments, as determined by searching the Taberogu website (https:// tabelog.com/en/), which is used as a restaurant guide all over Japan. This indicates that nomihodai has become prevalent among Japanese people. Also, izakaya establishments are the most popular place for drinking among college students (Kitada 2011). Although it is easy to imagine that college students use this service as a matter of course, no previous study has examined the influence of nomihodai on alcohol consumption among Japanese college students. If we could clarify the influence of nomihodai among Japanese college students, who should be considered a high-risk group, we could use the results to enhance students' awareness and change the current alcohol policies.

The purpose of this study was to assess the difference in the amount of alcohol consumption between nomihodai use and non-use states, and to clarify the association between nomihodai use and HED among college students, with the goal of developing effective interventions aimed at reducing the harms of alcohol use.

\section{Materials and Methods}

Study design and participants

A cross-sectional study was conduced by self-administered questionnaire. From December 2016 to March 2017, we sampled undergraduate and graduate students above the age of 20 at 35 colleges in the Kanto area of Japan. The Kanto area, which is located in the eastern part of Japan, consists of several prefectures including Tokyo. In this study, students who had experience with nomihodai use were included, and people under the age of 20 (who are prohibited from drinking by Japanese law) were excluded.

\section{Data collection}

In this study, we adopted the following procedure to ensure the representativeness of our sample. According to the Education, Culture, Sports, Science and Statistics Handbook of 2014 (Ministry of Education, Culture, Sports, Science and Technology-Japan 2014a), the number of college or university students was $2,868,872$ in 2013. To collect samples from various backgrounds, we determined that the target number for each department of a college was 30. Subjects were selected from each of seven prefectures, including Tokyo (Kanto). In Japan, there are three main types of institutions: national, public, and private. According to a basic survey of schools performed in 2014 (Ministry of Education, Culture, Sports, Science and Technology-Japan 2014b), the relative proportions of each type of institution were as follows: national institute, $10.9 \%$; public institute, $11.4 \%$; and private institute, $77.7 \%$. We selected departments based on this ratio while referring to the university ranking list. We mailed the dean of each department to request understanding of and cooperation with our study. This mailing included a document that explained the research outline, including the objectives, procedures, and ethical consideration of this research, along with a consent form and return envelope. Also, we requested that the dean notify us of the name and address of the person responsible for maintaining contact and distributing questionnaires. To reach our target number, we sent our requests to 160 deans of faculties. Ultimately, 35 departments consented to our proposal. A total of 1,030 questionnaires were delivered through a single responsible contact at each college.

The questionnaire addressed 1) frequency of drinking alcohol; 2) frequency of binge drinking in the past year, as evaluated by the following question: "During the last 12 months, how often did you have five or more (for males) or four or more (for females) drinks containing any kind of alcohol within a two-hour period?"; 3) nomihodai use: "Did you use nomihodai? (Yes or No)"; 4) the number of drinks consumed when using or not using nomihodai; and 5) sex and age for demographic data. To improve the accuracy of the reported number of drinks, subjects were required to list everything they drank and describe the number of drinks. Types of alcohol listed in the questionnaire included sake, beer, shochu (Japanese distilled beverage), chuhai (spirit-based cocktail), cocktail, plum wine, whiskey, and wine. To report the frequency of drinking, the subject chose one of the following answers: "every day," "six times a week," "five times a week," "four times a week," "three times a week," "twice a week," "once a week," "three times a month," "twice a month," "once a month," "3 to 11 times a year," "once or twice a year," and "less than once a year."

\section{Statistical analyses}

Characteristics of participants were compared between males and females. Furthermore, in both males and females, the amount of drinking and the proportion of HED were compared between nomihodai use states and non-use states. Paired t-test was used to compare the means of the two types of states. The Fisher's exact test 
was used to examine the significance of the distribution difference between the two types of states.

The level of statistical significance was set at $P<0.05$. All statistical analyses were performed using Stata 13.1 for Windows (Stata Corp., College Station, TX, USA).

\section{Ethical considerations}

This research was approved by the medical ethics committee of National Defense Medical College.

\section{Results}

During the study period, 594 students out of 1,030 responded (57.7\% response rate). After 22 were excluded due to lack of data, the remaining 533 were suitable for analysis; of them 511 (95.8\%) had experienced nomihodai use. In this study, the data from those 511 students were analyzed. A flow chart is shown in Fig. 1.

Characteristics of study participants are provided in Table 1. Subjects included 274 men and 237 women. Significant differences between men and women included frequency of drinking in a week (men: $1.5 \pm 1.7$ days; women: $1.0 \pm 1.3$ days), amount of drinking (men: $62.9 \pm$ $43.5 \mathrm{~g}$ /day; women: $46.3 \pm 32.9 \mathrm{~g} /$ day), and occurrence of binge drinking (men: $77.7 \%$ vs. women: $63.3 \%$ ).

The amount of drinking was increased 1.8-fold (85.9 \pm $49.7 \mathrm{~g}$ vs. $48.2 \pm 29.5 \mathrm{~g})$ among men and 1.7-fold (63.7 \pm $39.3 \mathrm{~g}$ vs. $36.5 \pm 26.7 \mathrm{~g}$ ) among women during nomihodai use, compared with non-use states (Fig. 2). Furthermore, 109 (39.8\%) men and 71 (30.3\%) women reported HED only at nomihodai states.

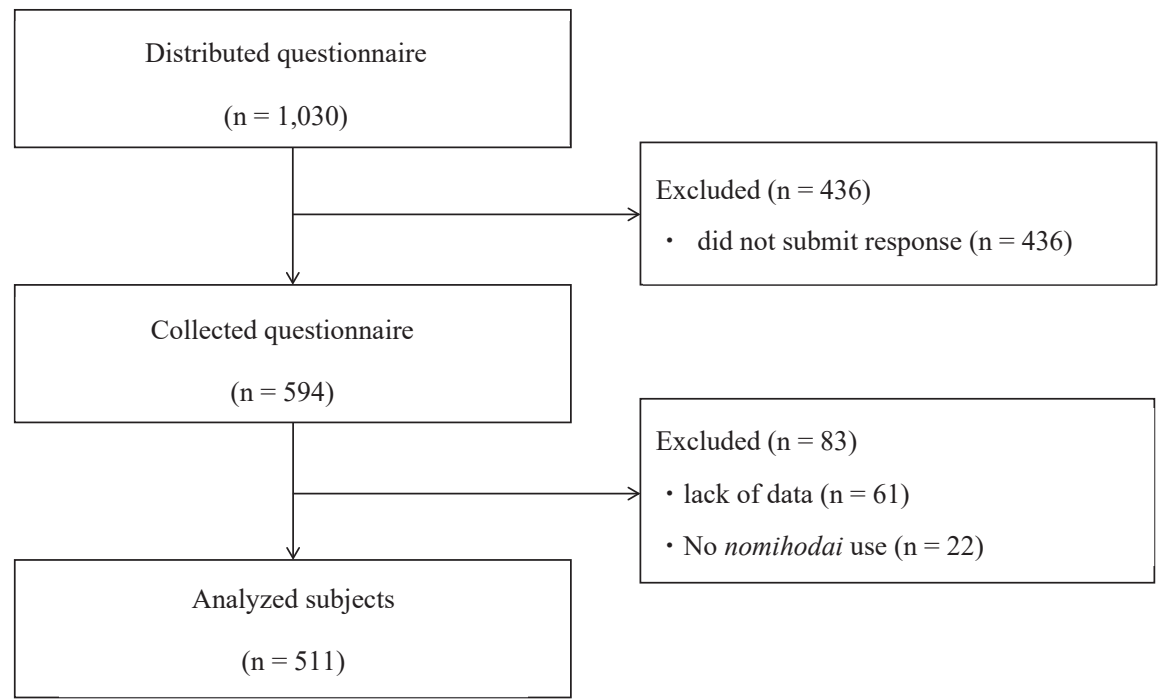

Fig. 1. Flow chart of subject selection for this study.

Table 1. Characteristics of participants.

\begin{tabular}{|c|c|c|c|c|}
\hline Variable & $\begin{array}{c}\text { Total } \\
(\mathrm{n}=511)\end{array}$ & $\begin{array}{c}\text { men } \\
(\mathrm{n}=274)\end{array}$ & $\begin{array}{c}\text { women } \\
(\mathrm{n}=237)\end{array}$ & $P$ \\
\hline Age (years), mean \pm SD & $21.7 \pm 1.9$ & $21.8 \pm 1.3$ & $21.6 \pm 2.5$ & $0.254^{\mathrm{b}}$ \\
\hline \multicolumn{5}{|c|}{ Frequency of drinking in one week } \\
\hline mean $\pm \mathrm{SD}$ & $1.2 \pm 1.5$ & $1.5 \pm 1.7$ & $1.0 \pm 1.3$ & $<0.001^{\mathrm{c}}$ \\
\hline \multicolumn{5}{|l|}{ Amount of drinking(g/day) } \\
\hline mean $\pm \mathrm{SD}$ & $55.2 \pm 39.8$ & $62.9 \pm 43.5$ & $46.3 \pm 32.9$ & $<0.001^{\mathrm{b}}$ \\
\hline \multicolumn{5}{|c|}{ Excessive weekly drinking ${ }^{\mathrm{a}}, \mathrm{n}(\%)$} \\
\hline Yes & $107(20.9)$ & $63(23.0)$ & $44(18.6)$ & $0.220^{\mathrm{d}}$ \\
\hline No & $404(79.1)$ & $211(77.0)$ & $193(81.4)$ & \\
\hline \multicolumn{5}{|l|}{ Binge drinking, $\mathrm{n}(\%)$} \\
\hline Yes & $363(71.0)$ & $213(77.7)$ & $150(63.3)$ & $<0.001^{\mathrm{d}}$ \\
\hline No & $148(29.0)$ & $61(22.3)$ & $87(36.7)$ & \\
\hline
\end{tabular}

aPure alcohol intake of $>140 \mathrm{~g}$ for men and $>70 \mathrm{~g}$ for women.

bPaired t-test (men vs. women).

'Mann-Whitney's U test.

${ }^{\mathrm{d}}$ Chi-squared test (men vs. women). 


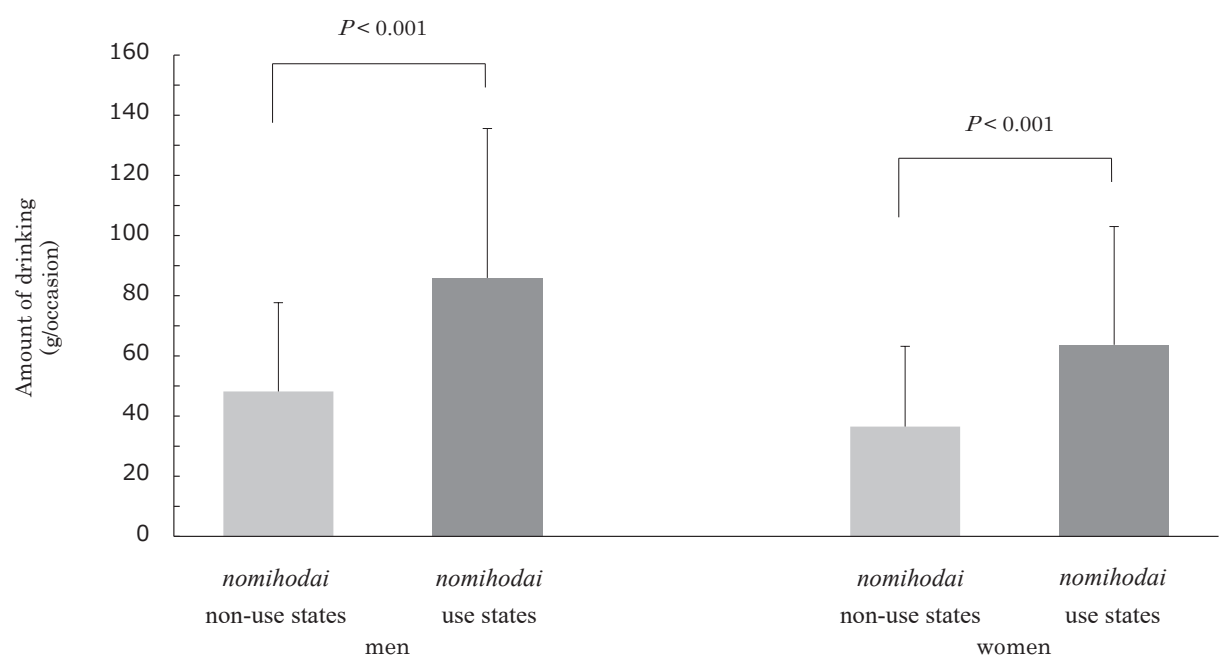

Fig. 2. Comparison of the amount of drinking during nomihodai use vs. non-use states.

\section{Discussion}

In this study, there was a significant difference in the amount of alcohol consumption between nomihodai use and non-use states. Also, approximately $60 \%$ of the students reported engaging in HED only when they used nomihodai, and during nomihodai use states 109 (39.8\%) men and 71 (30.3\%) women drank only in an HED manner.

Our study revealed that almost all college students had experience with nomihodai use. Nomihodai has become a common habit among Japanese people and is very prevalent. Accordingly, it is easy to imagine that college students use the service as a matter of course. We assume that these environmental factors contribute to the high ratio of nomihodai use among college students.

This study revealed that a significant difference in the amount of drinking between nomihodai use and non-use states. As mentioned above, previous studies reported that a set price for unlimited drinking is associated with HED among college students (Wechsler et al. 2000), and that there is a significant relationship between "all-you-candrink" and BAC (Thombs et al. 2009). Moreover, Thombs et al. (2009) reported that students who do not take advantage of drink specials (e.g., "all-you-can-drink") reported consuming more drinks before bar entry than those who did participate in these promotions. Thus, college students who use systems like "all-you-can-drink" try to make the most of the occasion so that they can thoroughly enjoy drinking.

Nomihodai establishments do not permit individual use; consequently, students always participate in nomihodai in groups. The presence of other students may influence the amount of consumed alcohol, based on two contributory factors. One is the "peer drinking norm," which involves recognition and approval of peers' drinking behavior, including drinking frequency and the amount of alcohol consumed (Borsari and Carey 2001). If students recognize that other students are drinking more, according to the peer drinking norm, it is possible that they will feel pressured to do the same. Particularly in occasions of nomihodai use, students who did not drink as much were often affected by other students who engaged in heavier drinking, and encouraged to make the most of the system. The peer drinking norm can have a positive effect, and it is important that we encourage behavioral change among students by providing information about actual peer drinking behavior and correcting their misunderstandings. Another factor is "peer pressure." Mekonen et al. (2017) indicated that peer pressure is an important predictor of problematic drinking among college students. If peer pressure is excessive, there is a risk that a student will insist that other students drink more. In Japan, the proportion of the students who have experienced being insisted by others to drink has been reported as 42\% (Masaki 2007), 57\% (Takase and Kawai 2010), and 47\% (Kawai et al. 2014), i.e., about half of all students. Furthermore, the study of Masaki (2007) revealed that $55.0 \%$ of students accepted that request, and an additional $31 \%$ drank a bit more, indicating that $86 \%$ of students did not refuse the insistence of another student to drink more, at least to some extent. It is necessary to educate students about how to enjoy the situation while understanding that some students have no or little alcohol tolerance due to a natural deficiency in acetaldehyde dehydrogenase. In addition, protective behavior can decrease risky drinking behavior among students who cannot or do not want to drink (Martens et al. 2011; Kim and Park 2015). We must support the ability of students to refuse the request to drink and to engage in self-protective behavior.

There were three limitations to this study. The first is the presence of geographical bias. The survey was conducted at 35 different colleges in the Kanto area in Japan. However, these institutions span a wide range of fields of study and included both private and public institutions, and the locations of the colleges encompass several prefectures, including both urban and rural areas. 
Therefore, geographic bias was minimized. A second limitation is sampling bias, based on the fact that distribution of questionnaire to students differed among colleges. The third possible limitation is recall bias. In this study, participants reported the amount of alcohol consumed and several experiences over the course of one year, using self-administered questionnaires. To grasp the actual amount of alcohol consumed, a future study might ask subjects to record their alcohol consumption using an "alcohol diary."

This study has revealed the significant influence of nomihodai on the amount of drinking among college students in Japan. Future efforts should promote preventive education and awareness activities that lead to safer alcohol-related behavior of college students.

\section{Acknowledgments}

This study was funded by JSPS KAKENHI Grant Number JP16K12362. The authors thank the deans of all 35 participating universities for helping with data collection.

\section{Conflict of Interest}

The authors declare no conflict of interest.

\section{References}

Borsari, B. \& Carey, K.B. (2001) Peer influences on college drinking: a review of research. J. Subs. Abuse, 13, 391-424.

Carlini, C., Andreoni, S., Martins, S.S., Benjamin, M., Sanudo, A. \& Sanchez, Z.M. (2014) Environmental characteristics associated with alcohol intoxication among patrons in Brazilian nightclubs. Drug Alcohol Rev., 33, 358-366.

Eaton, D.K., Kann, L., Kinchen, S., Shanklin, S., Ross, J., Hawkins, J., Harris, W.A., Lowry, R., McManus, T., Chyen, D., Lim, C., Whittle, L., Brener, N.D. \& Wechsler, H.; Centers for Disease Control and Prevention (CDC) (2010) Youth risk behavior surveillance-United States, 2009.

Higuchi, S., Matsushita, S., Maesato, H. \& Osaki, Y. (2007) Japan: alcohol today. Addiction, 102, 1849-1862.

Higuchi, S., Suzuki, K., Matsushita, S. \& Osaki, Y. (2004) Young people's drinking behavior in Japan.

https://www.researchgate.net/publication/237793695 [Accessed: March 4, 2018].

Hingson, R.W., Heeren, T., Zakocs, R.C., Kopstein, A. \& Wechsler, H. (2002) Magnitude of alcohol-related mortality and morbidity among U.S. college students ages 18-24. J. Stud. Alcohol, 63, 136-144.

Hingson, R.W., Zha, W. \& Weitzman, E.R. (2009) Magnitude of and alcohol-related mortality and morbidity among U.S. college students ages 18-24,1998-2005. J. Stud. Alcohol Drugs Suppl., 16, 136-144.

Kawai, S., Ohta, R., Negishi, R., Otomaru, T. \& Uemura, A. (2014) Attitudes of college students towards alcohol consumption: changes after five months of group living in a dormitory while abstaining from alcohol. Journal of health and supports science Juntendo University, 6, 22-26.

Kim, J. \& Park, S. (2015) Association between protective behavioral strategies and problem drinking among college students in the Republic of Korea. Addict. Behav., 51, 171-176.
Kitada, T. (2011) A study on the drinking behavior and attitude in college students. The Journal of Aichigakuin University, 59, 81-90.

Kowalenko, T., Burgess, B., Szpunar, S.M. \& Irvin-Babcock, C.B. (2013) Alcohol and trauma: in every age group. Am. J. Emerg. Med., 31, 705-709.

Martens, M.P., Martin, J.L., Littlefield, A.K., Murphy, J.G. \& Cimini, M.D. (2011) Changes in protective behavioral strategies and alcohol use among college students. Drug Alcohol Depend., 118, 504-507.

Masaki, M. (2007) Attitudes toward alcohol consumption at Hokudai: Japanese college students' awareness of drinking and alcohol use in their lives 2007. Bulletin of faculty of Education, Hokkaido University, 103, 113-126.

Mekonen, T., Fekadu, W., Chane, T. \& Bitew, S. (2017) Problematic Alcohol Use among University Students. Front. Psychiatry, 8, 86.

Ministry of Education, Culture, Sports, Science and TechnologyJapan (2014a) Education, Culture, Sports, Science and Statistics Handbook of 2014.

http://www.mext.go.jp/b_menu/toukei/002/002b/1349641.htm [Accessed: July 5, 2016].

Ministry of Education, Culture, Sports, Science and TechnologyJapan (2014b) Report on School Basic Survey of 2014: The number of higher education school.

http://www.mext.go.jp/component/b_menu/other/_icsFiles/ afieldfile/2014/12/19/1354124_1_1.pdf

[Accessed: July 5, 2016].

National Institute of Alcohol Abuse and Alcoholism (2004) NIAAA council approves definition of binge drinking. In NIAAA Newsletter: Winter, NIH Pulication, p. 3.

Osaki, Y., Kinjo, A., Higuchi, S., Matsumoto, H., Yuzuriha, T., Horie, Y., Kimura, M., Kanda, H. \& Yoshimoto, H. (2016) Prevalence and trends in alcohol dependence and alcohol use disorders in Japanese adults: results from periodical nationwide surveys. Alcohol Alcohol., 51, 465-473.

Takase, Y. \& Kawai, S. (2010) Attitudes of college students towards alcohol consumption at school of health and sport science, Juntendo University. Research Laboratory of Sports Medicine, School of Health and Sports Science, Juntendo University, 2, 106-112.

Thombs, D.L., O’Mara, R., Dodd, V.J., Hou, W., Merves, M.L., Weiler, R.M., Pokorny, S.B., Goldberger, B.A., Reingle, J. \& Werch, C.C. (2009) A field study of bar-sponsored drink specials and their associations with patron intoxication. $J$. Stud. Alcohol Drugs, 70, 206-214.

Wechsler, H., Kuo, M., Lee, H. \& Dowdall, G.W. (2000) Environmental correlates of underage alcohol use and related problems of college students. Am. J. Prev. Med., 19, 24-29.

White, A. \& Hingson, R. (2013) The burden of alcohol use: excessive alcohol consumption and related consequences among college students. Alcohol Res., 35, 201-218.

World Health Organization (WHO) (2009) Global Health Risks: Mortality and burden of disease attributable to selected major risk factors. World Health Organization, Geneva, Switzerland.

World Health Organization (WHO) (2014) Global status report on alcohol and health 2014. World Health Organization, Geneva, Switzerland.

Yoshimoto, H., Takayashiki, A., Goto, R., Saito, G., Kawaida, K., Hieda, R., Kataoka, Y., Aramaki, M., Sakamoto, N., Maeno, T., Kobayashi, Y. \& Takemura, Y.C. (2017) Association between excessive alcohol use and alcohol-related injuries in college students: multi-center cross-sectional study in Japan. Tohoku J. Exp. Med., 242, 157-163. 